\title{
Metal Coordination-Assisted Near-Infrared Photochromic Behavior - A Large Perturbation on Absorption Wavelength Properties of N,N-Donor Ligands Containing Diarylethene Derivatives by Coordination to Rhenium(I) Metal Center
}

Penny Ho-Man Lee, Chi-Chiu Ko, Nianyong Zhu, and Vivian Wing-Wah Yam*

Center for Carbon-Rich Molecular and Nano-Scale Metal-Based Materials Research, Department of Chemistry, and HKU-CAS Joint Laboratory on New Materials, The University of Hong Kong, Pokfulam Road, Hong Kong, PR China

\section{Supporting Information}




\section{Experimental Section:}

\section{Synthesis of $\mathbf{L 1}, \mathbf{L} 2$ and $\mathbf{L} 3$}

The ligands were synthesized by the bis-coupling reaction of 2-methylthien-3-ylboronic acid with 1-aryl-4,5-dibromo-2-(2-pyridyl)imidazole according to the standard Suzuki coupling procedure under an inert atmosphere of nitrogen. ${ }^{1}$ To a stirred solution of 1-aryl-4,5-dibromo-2-(2-pyridyl)imidazole (1 mmol), 2-methylthien-3-yl boronic acid $(2.2 \mathrm{mmol}), \mathrm{Pd}\left(\mathrm{PPh}_{3}\right)_{4}(0.1 \mathrm{mmol})$ in dioxane $(20 \mathrm{ml})$ was added aqueous $\mathrm{Cs}_{2} \mathrm{CO}_{3}$ solution $(2 \mathrm{M}, 5 \mathrm{ml}, 10 \mathrm{mmol})$. The resulting heterogeneous mixture was vigorously stirred and heated under reflux, and the course of reaction monitored by TLC. The resulting solution was then extracted three times with chloroform $(3 \times 50 \mathrm{ml})$, and the combined extracts were dried over anhydrous magnesium sulfate. After filtration and removal of the solvent under reduced pressure, the residue was purified by column chromatography on silica gel (70 - 230 mesh) using chloroform as eluent to give an analytically pure sample of the product as a white powder. Yield: 50 $60 \%$.

\section{Synthesis of $\mathbf{1}, 2$ and $\mathbf{3}$}

The complex was prepared by modification of a literature method for related $\operatorname{Re}(\mathrm{I})$ diimine complexes. ${ }^{2}\left[\mathrm{Re}(\mathrm{CO})_{5} \mathrm{Cl}\right](100 \mathrm{mg}, 0.27 \mathrm{mmol})$ and the corresponding ligand $(0.28 \mathrm{mmol})$ were suspended in benzene $(25 \mathrm{~mL})$. The suspension was heated to reflux for $10 \mathrm{~h}$ under nitrogen, during which the starting materials dissolved gradually to give a yellow solution. After removing the solvent under reduced pressure, the residue was purified by column chromatography on silica gel (70 - 230 mesh) with chloroform as eluent. Yellow crystals were obtained by slow diffusion of $n$-hexane into a concentrated dichloromethane solution of the complex. Yield: $80-90 \%$. 
Yield: $52 \%$. ${ }^{1} \mathrm{H}$ NMR (400 MHz, $\mathrm{CDCl}_{3}, 298 \mathrm{~K}$, relative to $\mathrm{Me}_{4} \mathrm{Si}$ ): $\delta 1.90$ (s, 3H, 2-Me of thiophene ring), $2.22(\mathrm{~s}, 3 \mathrm{H}, 2-\mathrm{Me}$ of thiophene ring), 2.28(s, 3H, 5-Me of thiophene ring), 2.35 (s, 3H, 5-Me of thiophene ring), $3.80(\mathrm{~s}, 1 \mathrm{H},-\mathrm{OMe}), 6.12(\mathrm{~s}, 1 \mathrm{H}$, thienyl proton at 4-position), $6.61(\mathrm{~s}, 1 \mathrm{H}$, thienyl proton at 4-position), $6.77(\mathrm{~d}, 2 \mathrm{H}$, phenyl proton at 2-position), $6.99(\mathrm{~d}, 2 \mathrm{H}$, phenyl proton at 3-position), 7.11 (td, 1H, pyridyl proton at 5-position), 7.65 (td, 1H, pyridyl proton at 3-position), 7.89 (d, $1 \mathrm{H}$, pyridyl proton at 5-position), $8.33(\mathrm{~d}, 1 \mathrm{H}$, pyridyl proton at 2-position). Positive-ion EI mass spectrum: $m / z 471\{\mathrm{M}\}^{+}, 456\left\{\mathrm{M}-\mathrm{CH}_{3}\right\}^{+}$. Elemental analyses, Found (\%): C 68.68, H 5.44, N 8.76; Calcd for L1 (\%): C 68.76, H 5.34, N: 8.91.

Characterization of 1-(4-Methylphenyl)-4,5-bis-[2,5-dimethyl-3-thienyl]-2-(2-pyridyl)imidazole (L2)

Yield: $54 \%$. ${ }^{1} \mathrm{H}$ NMR (400 MHz, $\mathrm{CDCl}_{3}, 298 \mathrm{~K}$, relative to $\mathrm{Me}_{4} \mathrm{Si}$ ): $\delta 1.88$ (s, 3H, 2-Me of thiophene ring), 2.17 (s, 3H, 2-Me of thiophene ring), 2.19(s, 3H, 5-Me of thiophene ring), 2.26 (s, 3H, 5-Me of thiophene ring), $2.36(\mathrm{~s}, 3 \mathrm{H}, 4-\mathrm{Me}$ of phenyl ring), $6.10(\mathrm{~s}, 1 \mathrm{H}$, thienyl proton at 4-position), 6.61 (s, $1 \mathrm{H}$, thienyl proton at 4-position), $6.92(\mathrm{~d}, 2 \mathrm{H}$, phenyl proton at 2-position), $7.03(\mathrm{~d}, 2 \mathrm{H}$, phenyl proton at 3-position), $7.11(\mathrm{td}, 1 \mathrm{H}$, pyridyl proton at 5-position), $7.65(\mathrm{td}, 1 \mathrm{H}$, pyridyl proton at 3-position), 7.88 (d, 1H, pyridyl proton at 5-position), 8.33 (d, 1H, pyridyl proton at 2-position). Positive-ion EI mass spectrum: $m / z 455\{\mathrm{M}\}^{+}, 440\left\{\mathrm{M}-\mathrm{CH}_{3}\right\}^{+}$. Elemental analyses, Found (\%): C 70.87, H 5.39, N 9.15; Calcd for L2 (\%): C 71.17, H 5.53, N: 9.22.

Characterization of N-Methyl-4,5-bis-[2,5-dimethyl-3-thienyl]-2-(2-pyridyl)imidazole (L3) 
Yield: $60 \%$. ${ }^{1} \mathrm{H}$ NMR (400 MHz, $\mathrm{CDCl}_{3}, 298 \mathrm{~K}$, relative to $\mathrm{Me}_{4} \mathrm{Si}$ ): $\delta 1.87$ (s, 3H, 2-Me of thiophene ring), 2.09 (s, 3H, 2-Me of thiophene ring), 2.12(s, 3H, 5-Me of thiophene ring), 2.20 (s, 3H, 5-Me of thiophene ring), $3.90(\mathrm{~s}, 1 \mathrm{H},-\mathrm{NMe}), 6.00(\mathrm{~s}, 1 \mathrm{H}$, thienyl proton at 4-position), $6.51(\mathrm{~s}, 1 \mathrm{H}$, thienyl proton at 4-position), $6.90(\mathrm{~d}, 2 \mathrm{H}$, phenyl proton at 2-position), $7.00(\mathrm{~d}, 2 \mathrm{H}$, phenyl proton at 3-position), 7.10 (td, 1H, pyridyl proton at 5-position), 7.62 (td, 1H, pyridyl proton at 3-position), 7.85 (d, $1 \mathrm{H}$, pyridyl proton at 5-position), $8.31(\mathrm{~d}, 1 \mathrm{H}$, pyridyl proton at 2-position). Positive-ion EI mass spectrum: $m / z 379\{\mathrm{M}\}^{+}, 364\left\{\mathrm{M}-\mathrm{CH}_{3}\right\}^{+}$. Elemental analyses, Found (\%): C 66.25, H 5.52, N 10.89; Calcd for L3 (\%): C 66.47, H 5.58, N: 11.08.

\section{Characterization of $[\operatorname{Re}(\mathrm{CO})(\mathbf{L 1}) \mathrm{Cl}](\mathbf{1})$}

Yield: $82 \%$. ${ }^{1} \mathrm{H}$ NMR (400 MHz, $\mathrm{CDCl}_{3}, 298 \mathrm{~K}$, relative to $\mathrm{Me}_{4} \mathrm{Si}$ ): $\delta 1.95$ (s, 3H, 2-Me of thiophene ring), $2.10(\mathrm{~s}, 3 \mathrm{H}, 2-\mathrm{Me}$ of thiophene ring), 2.23(s, 3H, 5-Me of thiophene ring), 2.43 (s, 3H, 5-Me of thiophene ring), $3.89(\mathrm{~s}, 1 \mathrm{H},-\mathrm{OMe}), 6.10(\mathrm{~s}, 1 \mathrm{H}$, thienyl proton at 4-position), $8.1(\mathrm{~s}, 1 \mathrm{H}$, thienyl proton at 4-position), $7.00(\mathrm{~d}, 2 \mathrm{H}$, phenyl proton at 2-position), $7.00(\mathrm{~d}, 2 \mathrm{H}$, phenyl proton at 3-position), $6.70(\mathrm{td}, 1 \mathrm{H}$, pyridyl proton at 5-position), 7.30 (td, $1 \mathrm{H}$, pyridyl proton at 3-position), 7.65 (d, 1H, pyridyl proton at 5-position), 9.05 (d, 1H, pyridyl proton at 2-position). Positive-ion FAB mass spectrum: $m / z 728\{\mathrm{M}\}^{+}$. Elemental analyses, Found (\%): C 46.55, H 3.05, N 6.03; Calcd for 1 (\%): C 46.35, H 3.08, N: 6.13 .

\section{Characterization of $[\operatorname{Re}(\mathrm{CO}) 3(\mathbf{L} 2) \mathrm{Cl}](2)$}

Yield: $86 \%$. 1H NMR (400 MHz, $\mathrm{CDCl}_{3}, 298 \mathrm{~K}$, relative to $\mathrm{Me}_{4} \mathrm{Si}$ ): $\delta 1.95$ (s, 3H, 2-Me of thiophene ring), $2.10(\mathrm{~s}, 3 \mathrm{H}, 2-\mathrm{Me}$ of thiophene ring), 2.21(s, 3H, 5-Me of thiophene ring), 2.43 (s, 3H, 5-Me of thiophene ring), $2.47(\mathrm{~s}, 3 \mathrm{H}, 4-\mathrm{Me}$ of phenyl ring), $6.10(\mathrm{~s}, 1 \mathrm{H}$, thienyl proton at 4-position), 8.01 (s, 
$1 \mathrm{H}$, thienyl proton at 4-position), $7.30(\mathrm{~d}, 2 \mathrm{H}$, phenyl proton at 2-position), $7.30(\mathrm{~d}, 2 \mathrm{H}$, phenyl proton at 3-position), $6.70(\mathrm{td}, 1 \mathrm{H}$, pyridyl proton at 5-position), $7.30(\mathrm{td}, 1 \mathrm{H}$, pyridyl proton at 3-position), $7.60(\mathrm{~d}, 1 \mathrm{H}$, pyridyl proton at 5-position), 9.05 (d, 1H, pyridyl proton at 2-position). Positive-ion FAB mass spectrum: $m / z 712\{\mathrm{M}\}^{+}$. Elemental analyses, Found (\%): C 47.50, H 3.27, N 5.59; Calcd for 2 (\%): C 47.32, H 3.30, N: 5.52.

Characterization of $[\operatorname{Re}(\mathrm{CO}) 3(\mathbf{L} 3) \mathrm{Cl}](3)$

Yield: 90 \%. $1 \mathrm{H} \mathrm{NMR} \mathrm{(400} \mathrm{MHz,} \mathrm{CDCl}_{3}, 298 \mathrm{~K}$, relative to $\mathrm{Me}_{4} \mathrm{Si}$ ): $\delta 1.95$ (s, 3H, 2-Me of thiophene ring), $2.10(\mathrm{~s}, 3 \mathrm{H}, 2-\mathrm{Me}$ of thiophene ring), 2.21(s, 3H, 5-Me of thiophene ring), 2.40 (s, 3H, 5-Me of thiophene ring), $4.00(\mathrm{~s}, 3 \mathrm{H},-\mathrm{NMe}), 6.5-7.0(\mathrm{br}, 2 \mathrm{H}$, thienyl proton), 7.41 (m, 1H, pyridyl proton), $8.02(\mathrm{~m}, 2 \mathrm{H}$, pyridyl proton), $9.11(\mathrm{~d}, 1 \mathrm{H}$, pyridyl proton at 2-position). Positive-ion FAB mass spectrum: $m / z 636\{\mathrm{M}\}^{+}$. Elemental analyses, Found (\%): C 42.09, H 3.15, N 6.30; Calcd for 3 (\%): C 42.06, H 3.08, N: 6.13. 
${ }^{1} \mathrm{H}$ NMR spectra were recorded either on a Bruker DPX-300 (300 MHz), or a Bruker AV400 (400 $\mathrm{MHz})$ at $298 \mathrm{~K}$ with chemical shifts $(\delta, \mathrm{ppm})$ relative to tetramethylsilane $\left(\mathrm{Me}_{4} \mathrm{Si}\right)$. Positive-ion fast atom bombardment (FAB) and electron impact (EI) mass spectra were recorded on a Finnigan MAT95 mass spectrometer. Elemental analyses of the new compounds were performed on a Carlo Erba 1106 elemental analyzer at the Institute of Chemistry, Chinese Academy of Sciences, Beijing.

UV-vis absorption spectra were recorded on a Hewlett-Packard 8452A diode array spectrophotometer. Photoirradiation was carried out using a $300 \mathrm{~W}$ Xe (ozone-free) lamp, and monochromatic light was obtained by passing the light through an Applied Photophysics F 3.4 monochromator. All measurements were conducted at $25{ }^{\circ} \mathrm{C}$. The UV-vis absorption spectral changes were obtained by recording the UV-vis absorption spectra of a stirred solution during the course of irradiation at selected wavelength with time intervals of $30 \mathrm{~s}$.

Steady-state emission and excitation spectra at room temperature were recorded on a Spex Fluorolog 111 spectrofluorometer. For solution emission and excitation spectra, samples were rigorously degassed with no fewer than four freeze-pump-thaw cycles prior to the measurements.

Chemical actinometry was employed for the photochemical quantum yield determination. ${ }^{3}$ Incident light intensities were taken from the average values measured just before and after each photolysis experiment using Reinecke's salt actinometry. ${ }^{3}$ In the determination of the photochemical quantum yield, the sample solutions were prepared at concentrations with absorbance slightly greater than 2.0 at 
the excitation wavelength. The quantum yield was determined at a small percentage of conversion by monitoring the initial rate of change of absorbance $(\Delta A / \Delta t)$ in the absorption maximum of the closed forms in the visible region. The quantum yield for the cycloreversion was determined with excitation wavelength at $510 \mathrm{~nm}$ rather than at $710 \mathrm{~nm}$ due to the limitation of our excitation source at the NIR region. Absorption spectral changes of complex 2 on alternate excitation at $350 \mathrm{~nm}$ and $610 \mathrm{~nm}$ over two cycles were shown in Figure S4. Owing to the extremely low quantum yields, the small absorbances at the excitation wavelength of $610 \mathrm{~nm}$ near the completion of the reaction, and hence the extremely long irradiation times required for the photocycloreversion reactions, the complete return of the absorbance to zero baseline was difficult to achieve. Both complexes do not show excellent fatigue resistance, probably with some photo-degradation after prolonged irradiation.

\section{References:}

1. Ko, C. C.; Kwok, W. M.; Yam, V. W. W.; Phillips, D. L. Chem. Eur. J. 2006, 12, 5840-5848.

2. Caspar, J. V.; Sullivan, B. P.; Meyer, T. J. Inorg. Chem. 1984, 23, 2104-2109.

3. (a) Murov, S. L. Handbook of Photochemistry, ed. Marcel Dekker, New York, 1973, P. 299 - 313.

(b) Kuhn, H. J.; Braslavsky, S. E.; Schmidt, R. Pure Appl. Chem. 2004, 76(12), 2105 - 2146. (c)

Wegner, E. E.; Adamson, A. W. J. Am. Chem. Soc. 1966, 88, 394 - 404. (d) Searle, G. H.; Bull, G. S.; House, D. A. J. Chem. Educ. 1989, 66, 605 - 608. (e) Szychliński, J.; Bilski, P.; Martuszewski, K.; Blażejowski, J. Analyst, 1989, 114, 739 - 741. 


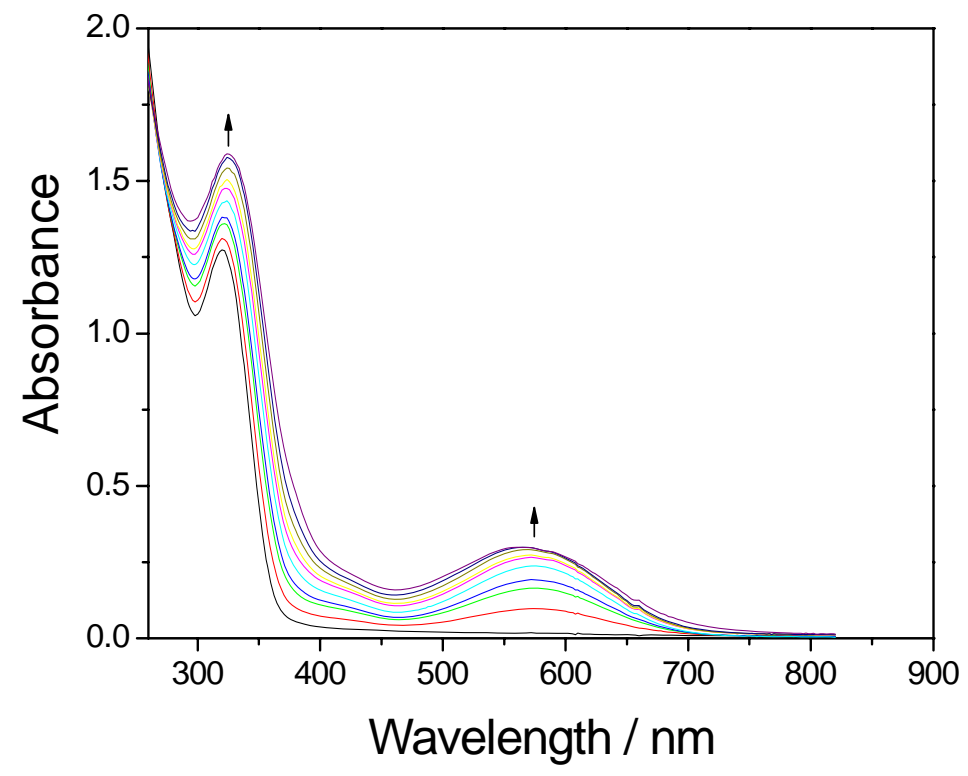

Figure S1 UV-vis absorption spectral changes of $\mathbf{L} 2$ in chloroform upon IL excitation at $320 \mathrm{~nm}$ at $298 \mathrm{~K}$.

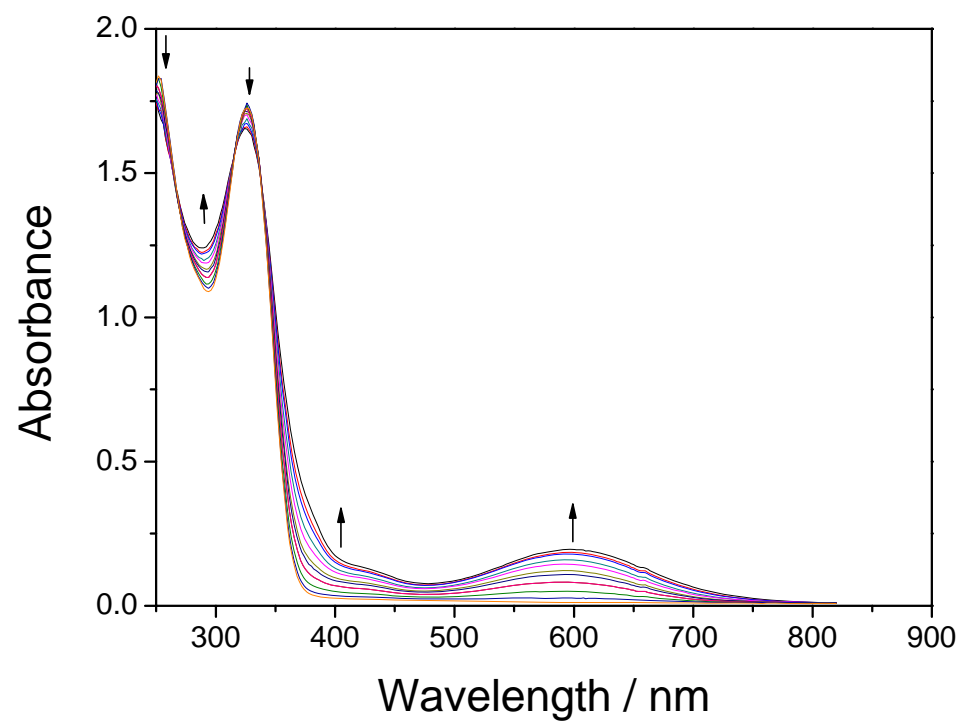

Figure S2 UV-vis absorption spectral changes of $\mathbf{L} 3$ in chloroform upon IL excitation at $320 \mathrm{~nm}$ at $298 \mathrm{~K}$. 


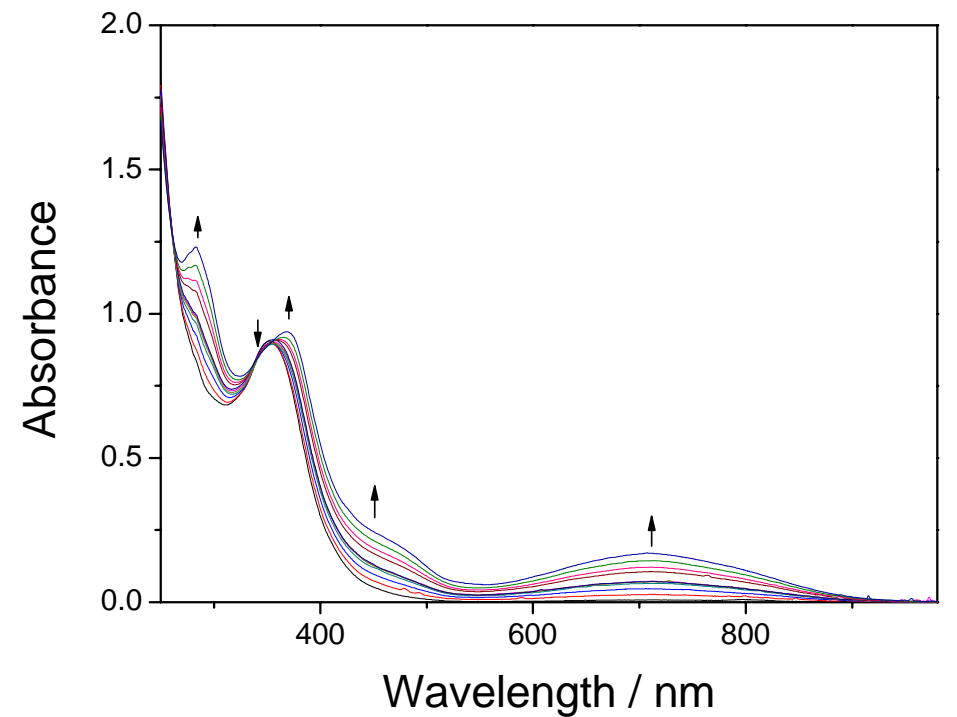

Figure S3 UV-vis absorption spectral changes of $\mathbf{1}$ in chloroform upon IL excitation at $320 \mathrm{~nm}$ at $298 \mathrm{~K}$.

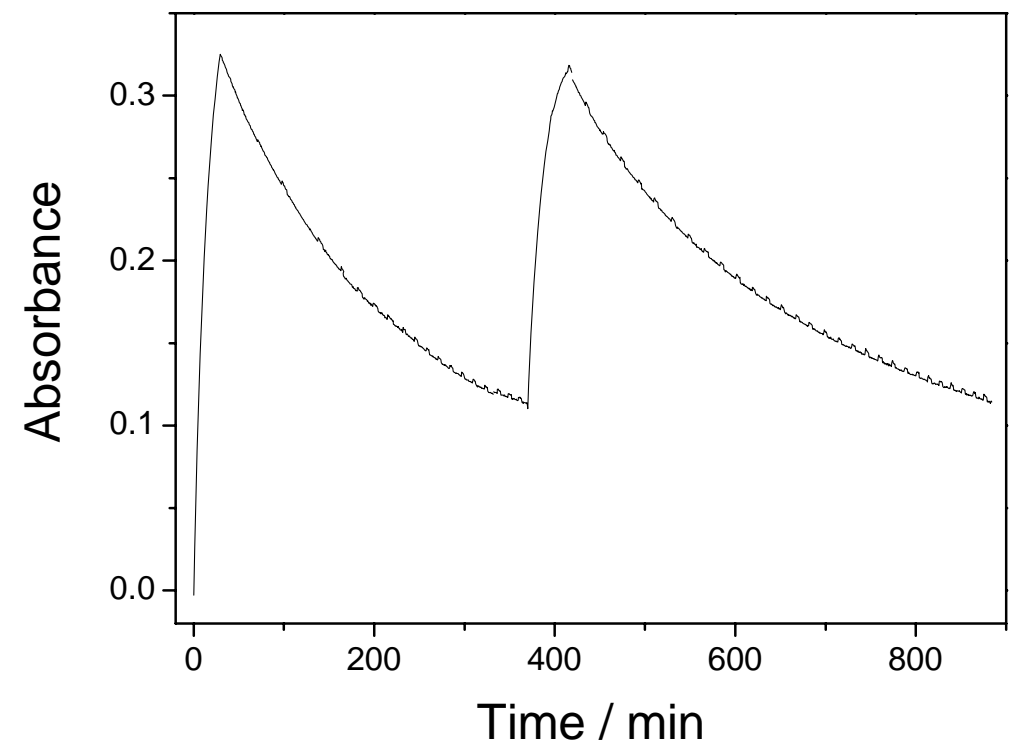

Figure S4 UV-vis absorption spectral changes of $2\left(1.2 \times 10^{-4} \mathrm{M}\right)$ at $710 \mathrm{~nm}$ on alternate excitation at $350 \mathrm{~nm}$ and $610 \mathrm{~nm}$ over two cycles in degassed chloroform solution at $288 \mathrm{~K}$. 


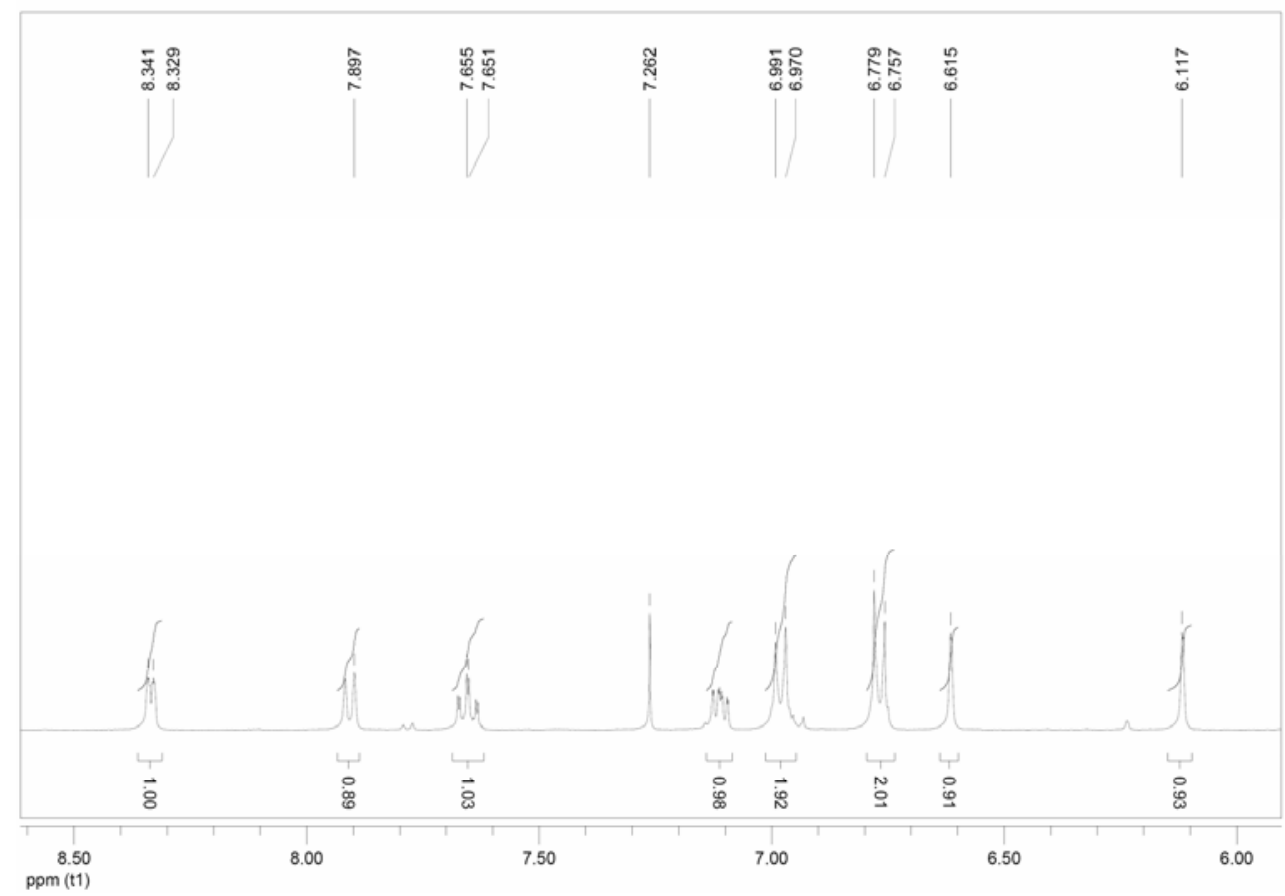

Figure S5 $\quad{ }^{1} \mathrm{H}$ NMR spectrum of $\mathbf{L 1}$ at $298 \mathrm{~K}$ in $\mathrm{CDCl}_{3}$ in aromatic region.

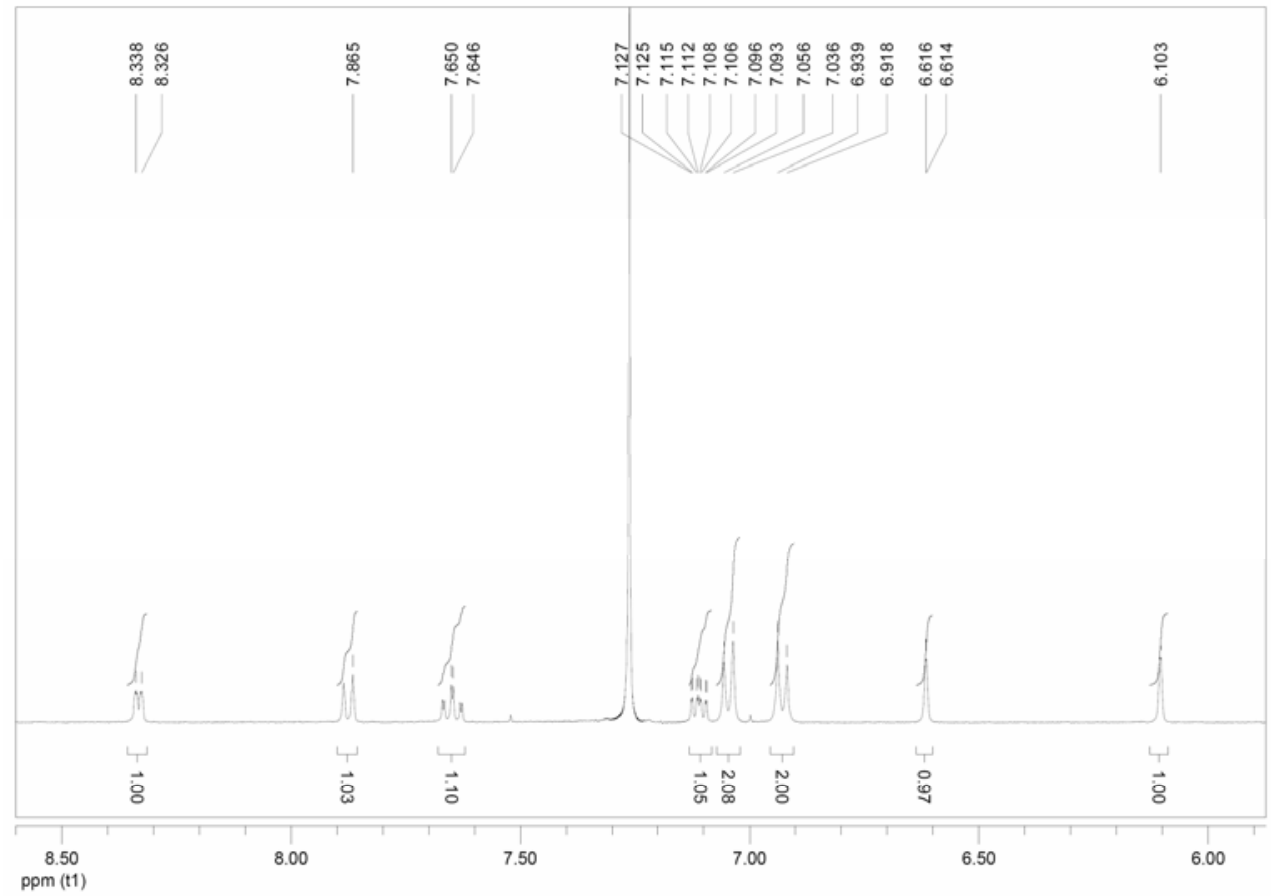

Figure S6 $\quad{ }^{1} \mathrm{H}$ NMR spectrum of $\mathbf{L} 2$ at $298 \mathrm{~K}$ in $\mathrm{CDCl}_{3}$ in aromatic region. 


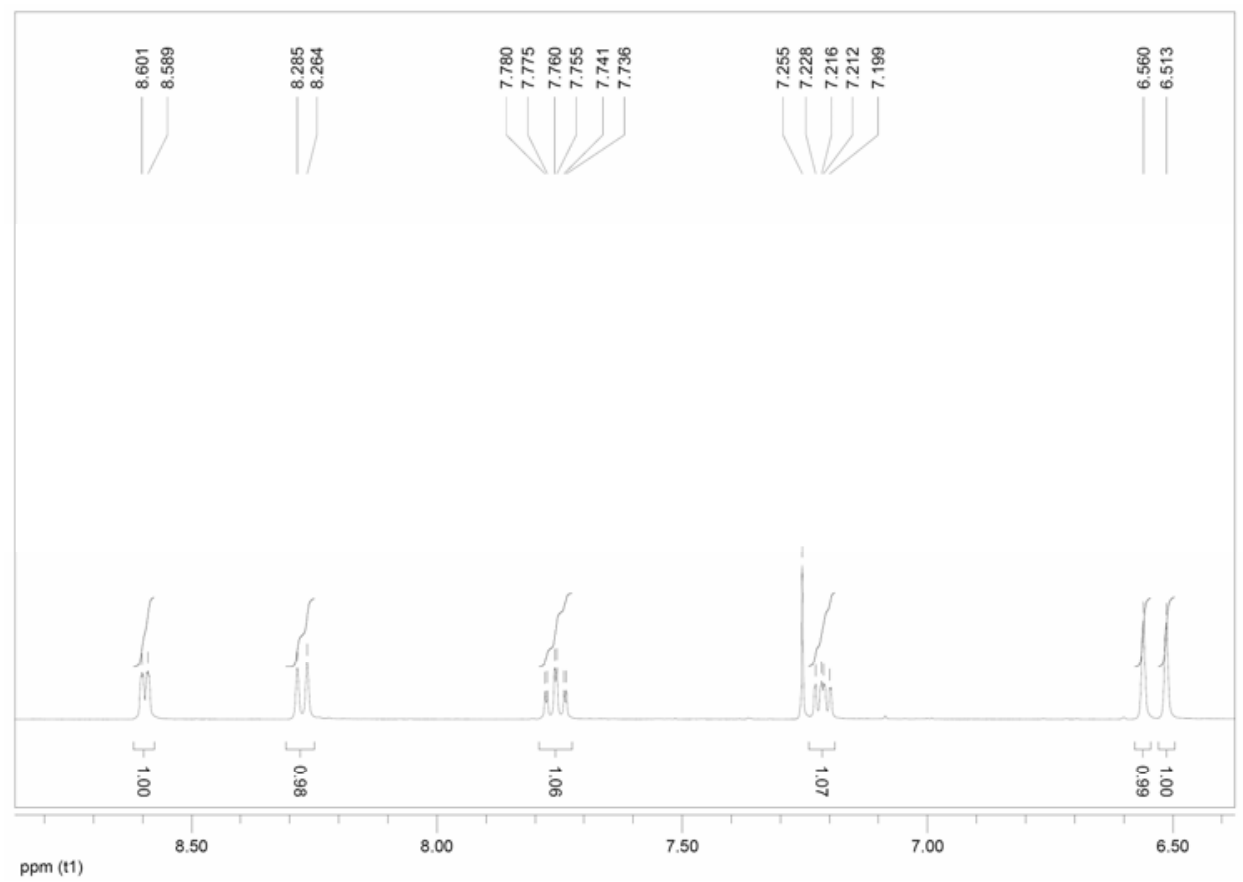

Figure S7 $\quad{ }^{1} \mathrm{H}$ NMR spectrum of $\mathbf{L 3}$ at $298 \mathrm{~K}$ in $\mathrm{CDCl}_{3}$ in aromatic region.

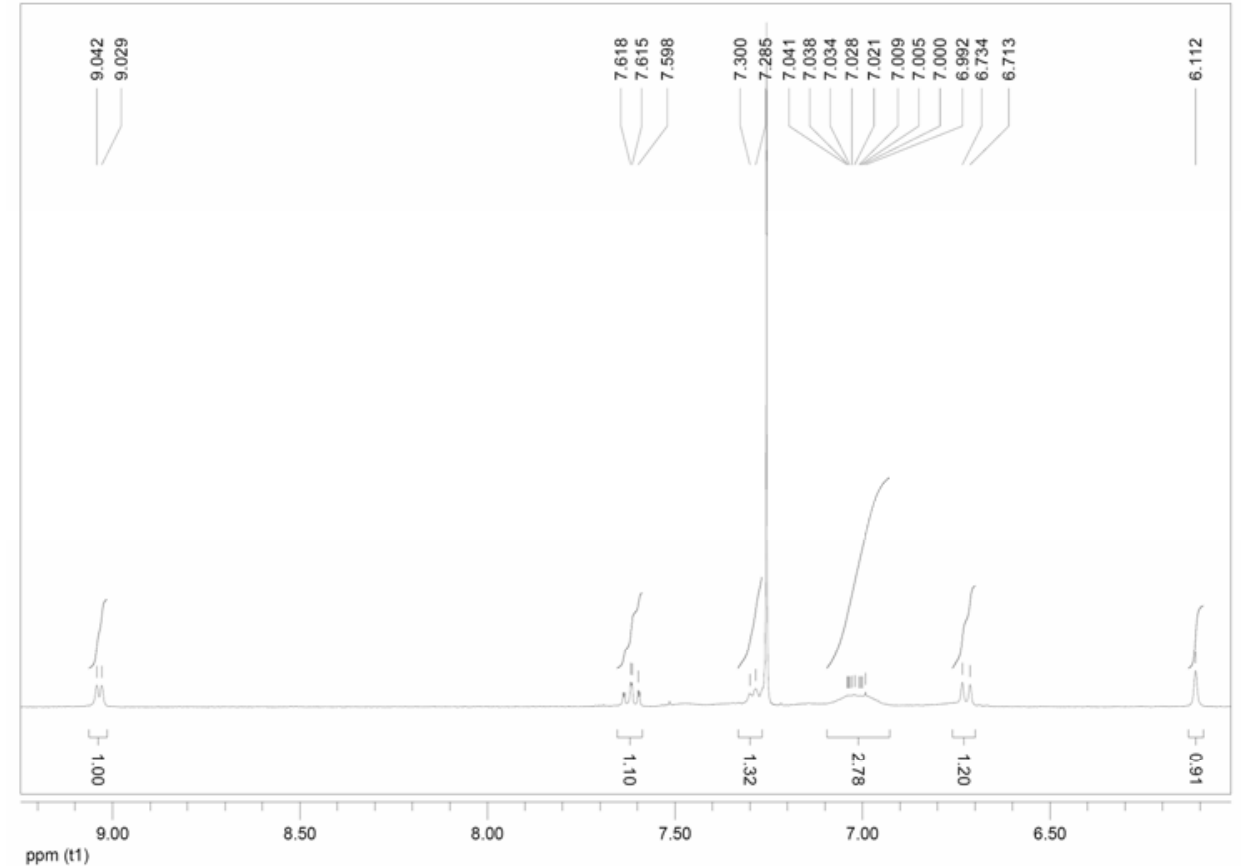

Figure S8 $\quad{ }^{1} \mathrm{H}$ NMR spectrum of $\mathbf{1}$ at $298 \mathrm{~K}$ in $\mathrm{CDCl}_{3}$ in aromatic region. 


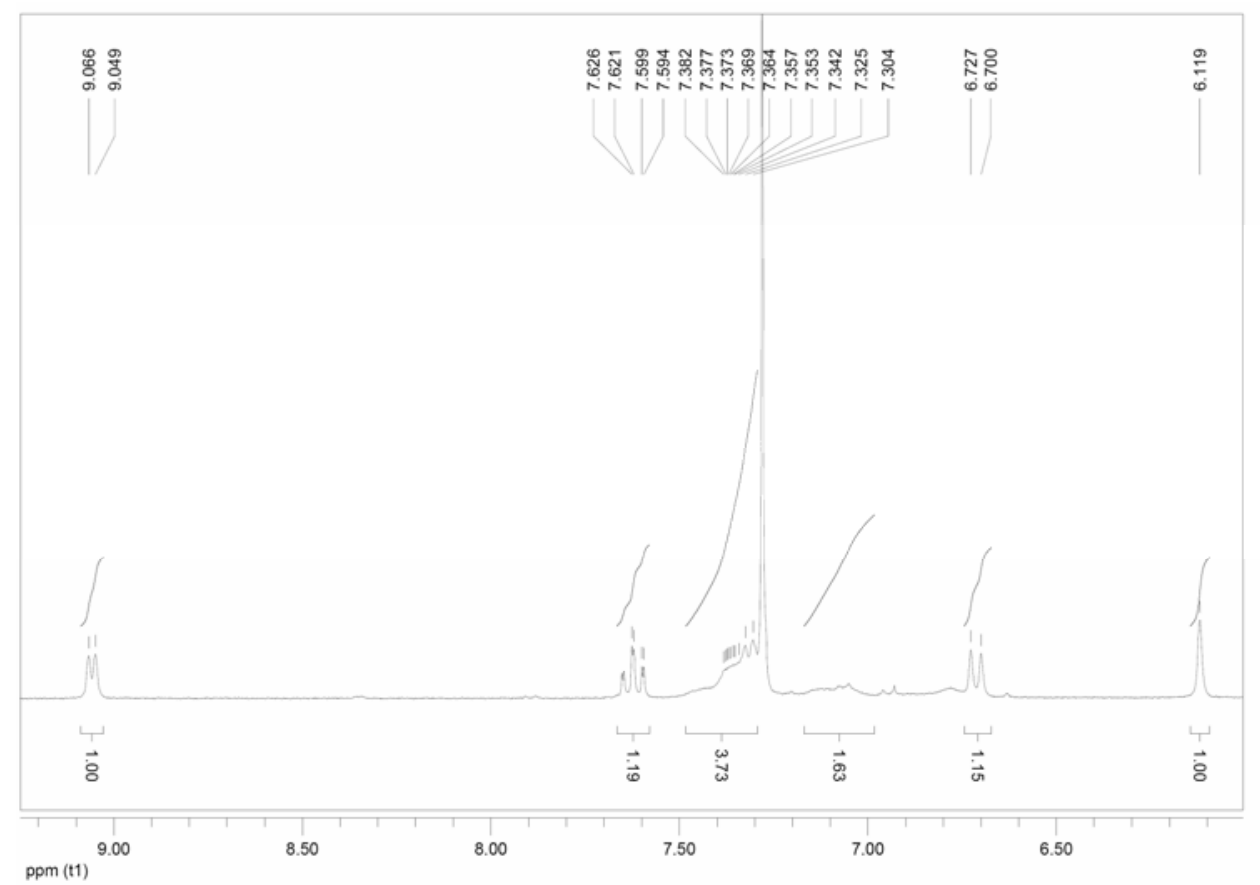

Figure S9 ${ }^{1} \mathrm{H}$ NMR spectrum of 2 at $298 \mathrm{~K}$ in $\mathrm{CDCl}_{3}$ in aromatic region.

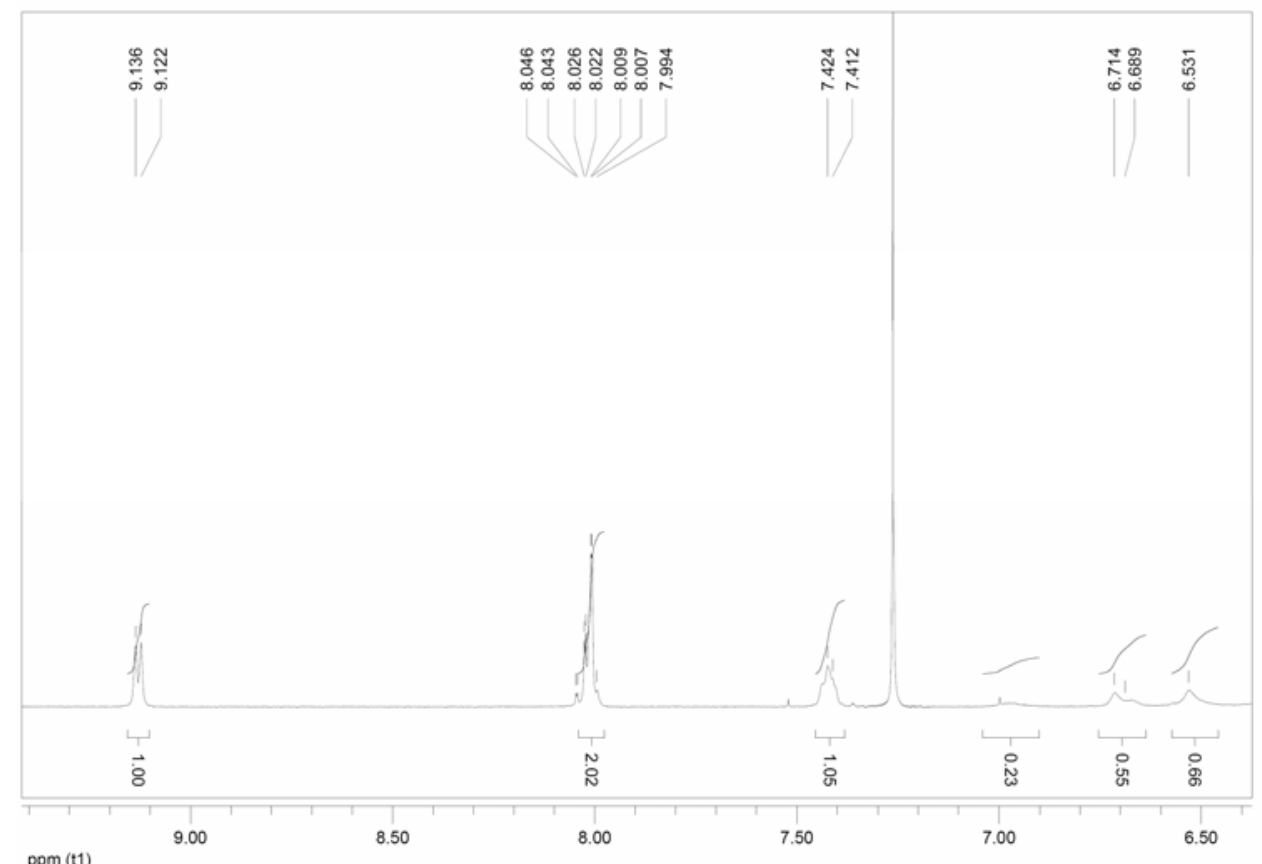

Figure S10 ${ }^{1} \mathrm{H}$ NMR spectrum of 3 at $298 \mathrm{~K}$ in $\mathrm{CDCl}_{3}$ in aromatic region. 
Table S1. Crystal data and structure refinement for complex 3.

Empirical formula

Formula weight, $M_{r}$

Temperature, $T / \mathrm{K}$

Wavelength, $\lambda / \AA$

Crystal system

Space group

Unit cell dimensions

Volume, $V / \AA^{3}$

$Z$

Density (calculated), $D_{c} / \mathrm{gcm}^{-3}$

Absorption coefficient

$F(000)$

Crystal dimension $/ \mathrm{mm}^{3}$

Theta range for data collection

Index ranges

Reflections collected

Independent reflections

Completeness to theta $=25.45^{\circ}$

Absorption correction

Refinement method

Data / restraints / parameters

Goodness-of-fit on $F^{2}$

Final $R$ indices $[I>2 \sigma(I)]$

$R$ indices (all data)

Residual extrema in final difference map, $\AA^{-3}$
$\mathrm{C}_{24} \mathrm{H}_{21} \mathrm{ClN}_{3} \mathrm{O}_{3} \mathrm{ReS}_{2}$

685.21

253(2)

0.71073

Monoclinic

$C 2 / c$

$a=23.414(5) \AA$

$\alpha=90^{\circ}$

$b=15.225(3) \AA$

$\beta=108.03(3)^{\circ}$

$c=15.071(3) \AA$

$\gamma=90^{\circ}$

$5108.7(18)$

8

1.782

$5.056 \mathrm{~mm}^{-1}$

2672

$0.4 \times 0.25 \times 0.15$

1.83 to $25.45^{\circ}$.

$-28 \leq \mathrm{h} \leq 27,-18 \leq \mathrm{k} \leq 18,-18 \leq 1 \leq 18$

16583

$4667[\mathrm{R}($ int $)=0.0725]$

$98.9 \%$

None

Full-matrix least-squares on $\mathrm{F}^{2}$

4667 / 0 / 312

0.580

$R_{1}=0.0272, w R_{2}=0.0588$

$R_{1}=0.0422, w R_{2}=0.0613$

0.882 and -1.361 
Table S2. Atomic coordinates $\left(\times 10^{4}\right)$ and equivalent isotropic displacement parameters $\left(\AA^{2} \times 10^{3}\right)$ for complex 3. $U(\mathrm{eq})$ is defined as one third of the trace of the orthogonalized $\mathrm{U}^{\mathrm{ij}}$ tensor.

\begin{tabular}{|c|c|c|c|c|}
\hline & $\mathrm{x}$ & $\mathrm{y}$ & $\mathrm{z}$ & $\mathrm{U}(\mathrm{eq})$ \\
\hline $\mathrm{Cl}(1)$ & $1364(1)$ & 774(1) & $6691(1)$ & $45(1)$ \\
\hline $\mathrm{O}(1)$ & $2453(2)$ & $2702(3)$ & $6632(3)$ & $64(1)$ \\
\hline $\mathrm{O}(3)$ & $642(2)$ & $3094(2)$ & $6862(3)$ & $55(1)$ \\
\hline$C(9)$ & $2495(2)$ & $-816(3)$ & $10272(3)$ & $44(1)$ \\
\hline$C(10)$ & $3980(3)$ & $3552(3)$ & $8124(5)$ & $80(2)$ \\
\hline$C(15)$ & $3637(3)$ & $67(3)$ & $7575(4)$ & $55(2)$ \\
\hline$C(16)$ & $4558(3)$ & $-2446(3)$ & $10435(4)$ & $56(2)$ \\
\hline $\mathrm{C}(22)$ & $2194(2)$ & $2429(3)$ & $7108(4)$ & $37(1)$ \\
\hline$C(24)$ & $1069(2)$ & $2684(3)$ & $7217(3)$ & $36(1)$ \\
\hline $\operatorname{Re}(1)$ & $1732(1)$ & $1965(1)$ & $7848(1)$ & $26(1)$ \\
\hline $\mathrm{S}(1)$ & $4005(1)$ & $1812(1)$ & $7513(1)$ & $49(1)$ \\
\hline $\mathrm{S}(2)$ & $4741(1)$ & $-643(1)$ & $10876(1)$ & $41(1)$ \\
\hline $\mathrm{O}(2)$ & $2247(2)$ & $3425(3)$ & $9236(3)$ & $65(1)$ \\
\hline $\mathrm{N}(1)$ & $1224(2)$ & $1233(2)$ & $8619(2)$ & $30(1)$ \\
\hline $\mathrm{N}(2)$ & $2351(2)$ & $1001(2)$ & $8672(2)$ & $25(1)$ \\
\hline $\mathrm{N}(3)$ & $2553(2)$ & $-141(2)$ & $9620(2)$ & $25(1)$ \\
\hline$C(1)$ & $656(2)$ & 1391(3) & $8560(3)$ & $43(1)$ \\
\hline $\mathrm{C}(2)$ & $315(2)$ & $824(4)$ & 8891(4) & $50(1)$ \\
\hline$C(3)$ & $570(3)$ & $61(4)$ & $9311(4)$ & $56(2)$ \\
\hline$C(4)$ & $1159(2)$ & $-112(3)$ & $9400(3)$ & $45(1)$ \\
\hline$C(5)$ & 1491(2) & $486(3)$ & $9063(3)$ & $31(1)$ \\
\hline$C(6)$ & $2121(2)$ & $416(3)$ & $9118(3)$ & $24(1)$ \\
\hline$C(7)$ & $2952(2)$ & $820(3)$ & $8871(3)$ & $25(1)$ \\
\hline$C(8)$ & $3087(2)$ & $107(3)$ & $9471(3)$ & $26(1)$ \\
\hline $\mathrm{C}(11)$ & $3800(2)$ & $2601(3)$ & $8157(4)$ & $39(1)$ \\
\hline$C(12)$ & $3454(2)$ & $2247(3)$ & $8635(3)$ & $36(1)$ \\
\hline$C(13)$ & $3345(2)$ & $1332(3)$ & $8480(3)$ & $26(1)$ \\
\hline$C(14)$ & $3624(2)$ & $998(3)$ & $7880(3)$ & $33(1)$ \\
\hline $\mathrm{C}(17)$ & $4312(2)$ & $-1523(3)$ & $10343(3)$ & $37(1)$ \\
\hline$C(18)$ & $3755(2)$ & $-1249(3)$ & $9874(3)$ & $33(1)$ \\
\hline$C(19)$ & $3660(2)$ & $-328(3)$ & $9930(3)$ & $26(1)$ \\
\hline
\end{tabular}




\begin{tabular}{lrrrr}
$\mathrm{C}(20)$ & $4163(2)$ & $90(3)$ & $10458(3)$ & $30(1)$ \\
$\mathrm{C}(21)$ & $4255(2)$ & $1042(3)$ & $10696(4)$ & $43(1)$ \\
$\mathrm{C}(23)$ & $2044(2)$ & $2869(3)$ & $8756(4)$ & $39(1)$ \\
\hline
\end{tabular}


Table S3. Bond lengths $[\AA]$ and angles $\left[{ }^{\circ}\right]$ for complex 3.

\begin{tabular}{|c|c|}
\hline $\mathrm{Cl}(1)-\operatorname{Re}(1)$ & $2.4778(13)$ \\
\hline $\mathrm{O}(1)-\mathrm{C}(22)$ & $1.151(5)$ \\
\hline $\mathrm{O}(3)-\mathrm{C}(24)$ & $1.159(5)$ \\
\hline $\mathrm{C}(9)-\mathrm{N}(3)$ & $1.456(5)$ \\
\hline $\mathrm{C}(10)-\mathrm{C}(11)$ & $1.514(7)$ \\
\hline$C(15)-C(14)$ & $1.493(6)$ \\
\hline$C(16)-C(17)$ & $1.508(6)$ \\
\hline$C(22)-\operatorname{Re}(1)$ & $1.915(6)$ \\
\hline $\mathrm{C}(24)-\operatorname{Re}(1)$ & $1.897(5)$ \\
\hline $\operatorname{Re}(1)-C(23)$ & $1.919(6)$ \\
\hline $\operatorname{Re}(1)-\mathrm{N}(2)$ & $2.164(3)$ \\
\hline $\operatorname{Re}(1)-\mathrm{N}(1)$ & $2.205(3)$ \\
\hline$S(1)-C(11)$ & $1.704(5)$ \\
\hline$S(1)-C(14)$ & $1.715(4)$ \\
\hline$S(2)-C(20)$ & $1.716(4)$ \\
\hline$S(2)-C(17)$ & $1.718(5)$ \\
\hline $\mathrm{O}(2)-\mathrm{C}(23)$ & $1.119(5)$ \\
\hline $\mathrm{N}(1)-\mathrm{C}(1)$ & $1.326(6)$ \\
\hline $\mathrm{N}(1)-\mathrm{C}(5)$ & $1.369(5)$ \\
\hline $\mathrm{N}(2)-\mathrm{C}(6)$ & $1.326(5)$ \\
\hline $\mathrm{N}(2)-\mathrm{C}(7)$ & $1.375(5)$ \\
\hline $\mathrm{N}(3)-\mathrm{C}(6)$ & $1.358(5)$ \\
\hline $\mathrm{N}(3)-\mathrm{C}(8)$ & $1.390(5)$ \\
\hline$C(1)-C(2)$ & $1.371(6)$ \\
\hline$C(2)-C(3)$ & $1.368(7)$ \\
\hline$C(3)-C(4)$ & $1.370(6)$ \\
\hline$C(4)-C(5)$ & $1.392(6)$ \\
\hline$C(5)-C(6)$ & $1.454(6)$ \\
\hline$C(7)-C(8)$ & $1.385(5)$ \\
\hline$C(7)-C(13)$ & $1.461(6)$ \\
\hline $\mathrm{C}(8)-\mathrm{C}(19)$ & $1.464(6)$ \\
\hline $\mathrm{C}(11)-\mathrm{C}(12)$ & $1.350(6)$ \\
\hline $\mathrm{C}(12)-\mathrm{C}(13)$ & $1.424(6)$ \\
\hline
\end{tabular}




\begin{tabular}{|c|c|}
\hline$C(13)-C(14)$ & $1.366(6)$ \\
\hline $\mathrm{C}(17)-\mathrm{C}(18)$ & $1.344(6)$ \\
\hline $\mathrm{C}(18)-\mathrm{C}(19)$ & $1.426(6)$ \\
\hline$C(19)-C(20)$ & $1.358(6)$ \\
\hline$C(20)-C(21)$ & $1.493(6)$ \\
\hline $\mathrm{O}(1)-\mathrm{C}(22)-\operatorname{Re}(1)$ & $177.3(5)$ \\
\hline $\mathrm{O}(3)-\mathrm{C}(24)-\operatorname{Re}(1)$ & $175.4(4)$ \\
\hline $\mathrm{C}(24)-\operatorname{Re}(1)-\mathrm{C}(22)$ & $90.99(19)$ \\
\hline $\mathrm{C}(24)-\mathrm{Re}(1)-\mathrm{C}(23)$ & $90.9(2)$ \\
\hline $\mathrm{C}(22)-\operatorname{Re}(1)-\mathrm{C}(23)$ & $89.5(2)$ \\
\hline $\mathrm{C}(24)-\mathrm{Re}(1)-\mathrm{N}(2)$ & $167.95(17)$ \\
\hline $\mathrm{C}(22)-\mathrm{Re}(1)-\mathrm{N}(2)$ & $100.68(16)$ \\
\hline $\mathrm{C}(23)-\mathrm{Re}(1)-\mathrm{N}(2)$ & $92.13(17)$ \\
\hline $\mathrm{C}(24)-\mathrm{Re}(1)-\mathrm{N}(1)$ & $93.73(17)$ \\
\hline $\mathrm{C}(22)-\mathrm{Re}(1)-\mathrm{N}(1)$ & $171.25(16)$ \\
\hline $\mathrm{C}(23)-\mathrm{Re}(1)-\mathrm{N}(1)$ & $97.80(18)$ \\
\hline $\mathrm{N}(2)-\operatorname{Re}(1)-\mathrm{N}(1)$ & $74.31(13)$ \\
\hline $\mathrm{C}(24)-\mathrm{Re}(1)-\mathrm{Cl}(1)$ & $91.01(15)$ \\
\hline $\mathrm{C}(22)-\mathrm{Re}(1)-\mathrm{Cl}(1)$ & $89.78(15)$ \\
\hline $\mathrm{C}(23)-\mathrm{Re}(1)-\mathrm{Cl}(1)$ & $177.95(14)$ \\
\hline $\mathrm{N}(2)-\mathrm{Re}(1)-\mathrm{Cl}(1)$ & $86.13(10)$ \\
\hline $\mathrm{N}(1)-\mathrm{Re}(1)-\mathrm{Cl}(1)$ & $82.78(10)$ \\
\hline $\mathrm{C}(11)-\mathrm{S}(1)-\mathrm{C}(14)$ & $93.4(2)$ \\
\hline$C(20)-S(2)-C(17)$ & $93.2(2)$ \\
\hline $\mathrm{C}(1)-\mathrm{N}(1)-\mathrm{C}(5)$ & $118.7(4)$ \\
\hline $\mathrm{C}(1)-\mathrm{N}(1)-\operatorname{Re}(1)$ & $124.5(3)$ \\
\hline $\mathrm{C}(5)-\mathrm{N}(1)-\operatorname{Re}(1)$ & $115.9(3)$ \\
\hline $\mathrm{C}(6)-\mathrm{N}(2)-\mathrm{C}(7)$ & $107.8(3)$ \\
\hline $\mathrm{C}(6)-\mathrm{N}(2)-\operatorname{Re}(1)$ & $116.2(3)$ \\
\hline $\mathrm{C}(7)-\mathrm{N}(2)-\operatorname{Re}(1)$ & $136.0(3)$ \\
\hline $\mathrm{C}(6)-\mathrm{N}(3)-\mathrm{C}(8)$ & $107.0(3)$ \\
\hline $\mathrm{C}(6)-\mathrm{N}(3)-\mathrm{C}(9)$ & $127.4(4)$ \\
\hline $\mathrm{C}(8)-\mathrm{N}(3)-\mathrm{C}(9)$ & $125.3(4)$ \\
\hline $\mathrm{N}(1)-\mathrm{C}(1)-\mathrm{C}(2)$ & $123.2(5)$ \\
\hline$C(3)-C(2)-C(1)$ & $118.8(5)$ \\
\hline
\end{tabular}




\begin{tabular}{ll}
$\mathrm{C}(2)-\mathrm{C}(3)-\mathrm{C}(4)$ & $119.5(5)$ \\
$\mathrm{C}(3)-\mathrm{C}(4)-\mathrm{C}(5)$ & $119.8(5)$ \\
$\mathrm{N}(1)-\mathrm{C}(5)-\mathrm{C}(4)$ & $119.9(4)$ \\
$\mathrm{N}(1)-\mathrm{C}(5)-\mathrm{C}(6)$ & $113.0(4)$ \\
$\mathrm{C}(4)-\mathrm{C}(5)-\mathrm{C}(6)$ & $127.0(4)$ \\
$\mathrm{N}(2)-\mathrm{C}(6)-\mathrm{N}(3)$ & $110.6(4)$ \\
$\mathrm{N}(2)-\mathrm{C}(6)-\mathrm{C}(5)$ & $119.0(4)$ \\
$\mathrm{N}(3)-\mathrm{C}(6)-\mathrm{C}(5)$ & $130.2(4)$ \\
$\mathrm{N}(2)-\mathrm{C}(7)-\mathrm{C}(8)$ & $108.0(4)$ \\
$\mathrm{N}(2)-\mathrm{C}(7)-\mathrm{C}(13)$ & $122.4(4)$ \\
$\mathrm{C}(8)-\mathrm{C}(7)-\mathrm{C}(13)$ & $129.5(4)$ \\
$\mathrm{C}(7)-\mathrm{C}(8)-\mathrm{N}(3)$ & $106.6(4)$ \\
$\mathrm{C}(7)-\mathrm{C}(8)-\mathrm{C}(19)$ & $130.8(4)$ \\
$\mathrm{N}(3)-\mathrm{C}(8)-\mathrm{C}(19)$ & $122.6(4)$ \\
$\mathrm{C}(12)-\mathrm{C}(11)-\mathrm{C}(10)$ & $128.2(5)$ \\
$\mathrm{C}(12)-\mathrm{C}(11)-\mathrm{S}(1)$ & $110.1(4)$ \\
$\mathrm{C}(10)-\mathrm{C}(11)-\mathrm{S}(1)$ & $121.6(4)$ \\
$\mathrm{C}(11)-\mathrm{C}(12)-\mathrm{C}(13)$ & $114.1(4)$ \\
$\mathrm{C}(14)-\mathrm{C}(13)-\mathrm{C}(12)$ & $112.0(4)$ \\
$\mathrm{C}(14)-\mathrm{C}(13)-\mathrm{C}(7)$ & $123.9(4)$ \\
$\mathrm{C}(12)-\mathrm{C}(13)-\mathrm{C}(7)$ & $124.0(4)$ \\
$\mathrm{C}(13)-\mathrm{C}(14)-\mathrm{C}(15)$ & $128.0(4)$ \\
$\mathrm{C}(13)-\mathrm{C}(14)-\mathrm{S}(1)$ & $110.3(3)$ \\
$\mathrm{C}(15)-\mathrm{C}(14)-\mathrm{S}(1)$ & $121.6(3)$ \\
$\mathrm{C}(18)-\mathrm{C}(17)-\mathrm{C}(16)$ & $128.2(5)$ \\
$\mathrm{C}(18)-\mathrm{C}(17)-\mathrm{S}(2)$ & $109.6(3)$ \\
$\mathrm{C}(16)-\mathrm{C}(17)-\mathrm{S}(2)$ & $122.2(4)$ \\
$\mathrm{C}(17)-\mathrm{C}(18)-\mathrm{C}(19)$ & $114.7(4)$ \\
$\mathrm{C}(20)-\mathrm{C}(19)-\mathrm{C}(18)$ & $111.9(4)$ \\
$\mathrm{C}(20)-\mathrm{C}(19)-\mathrm{C}(8)$ & $124.5(4)$ \\
$\mathrm{C}(18)-\mathrm{C}(19)-\mathrm{C}(8)$ & $123.6(4)$ \\
$\mathrm{C}(19)-\mathrm{C}(20)-\mathrm{C}(21)$ & $129.1(4)$ \\
$\mathrm{C}(19)-\mathrm{C}(20)-\mathrm{S}(2)$ & $110.6(3)$ \\
$\mathrm{C}(21)-\mathrm{C}(20)-\mathrm{S}(2)$ & $120.3(4)$ \\
$\mathrm{O}(2)-\mathrm{C}(23)-\mathrm{Re}(1)$ & $174.4(5)$ \\
\hline &
\end{tabular}


Table S4. Anisotropic displacement parameters $\left(\AA^{2} \times 10^{3}\right)$ for complex 3. The anisotropic displacement factor exponent takes the form: $-2 \pi^{2}\left[h^{2} a^{* 2} U^{11}+\ldots+2 h k a * b * U^{12}\right]$

\begin{tabular}{|c|c|c|c|c|c|c|}
\hline & $\mathrm{U}^{11}$ & $\mathrm{U}^{22}$ & $\mathrm{U}^{33}$ & $\mathrm{U}^{23}$ & $\mathrm{U}^{13}$ & $\mathrm{U}^{12}$ \\
\hline $\mathrm{Cl}(1)$ & $46(1)$ & $44(1)$ & $43(1)$ & $-12(1)$ & $12(1)$ & $-4(1)$ \\
\hline $\mathrm{O}(1)$ & $44(3)$ & $95(3)$ & $55(3)$ & $36(2)$ & $21(2)$ & $4(2)$ \\
\hline $\mathrm{O}(3)$ & $40(2)$ & $55(2)$ & $65(3)$ & $16(2)$ & $8(2)$ & $20(2)$ \\
\hline $\mathrm{C}(9)$ & $39(3)$ & $44(3)$ & $49(3)$ & $25(3)$ & $15(3)$ & $9(3)$ \\
\hline$C(10)$ & $69(5)$ & $37(3)$ & $134(7)$ & $14(4)$ & $31(5)$ & $-2(3)$ \\
\hline$C(15)$ & $70(4)$ & $44(3)$ & $59(4)$ & $-6(3)$ & $33(3)$ & $6(3)$ \\
\hline$C(16)$ & $59(4)$ & $37(3)$ & $69(4)$ & $2(3)$ & $16(3)$ & $19(3)$ \\
\hline$C(22)$ & $27(3)$ & $33(3)$ & $43(3)$ & $7(3)$ & $0(3)$ & $7(2)$ \\
\hline$C(24)$ & $34(3)$ & $36(3)$ & $39(3)$ & $-6(2)$ & $14(3)$ & $-5(2)$ \\
\hline $\operatorname{Re}(1)$ & $26(1)$ & $24(1)$ & $25(1)$ & $1(1)$ & $6(1)$ & $4(1)$ \\
\hline $\mathrm{S}(1)$ & $47(1)$ & $49(1)$ & $59(1)$ & $18(1)$ & $29(1)$ & $5(1)$ \\
\hline $\mathrm{S}(2)$ & $28(1)$ & $41(1)$ & $50(1)$ & $7(1)$ & $6(1)$ & $7(1)$ \\
\hline $\mathrm{O}(2)$ & $58(3)$ & $65(3)$ & $63(3)$ & $-27(2)$ & $5(2)$ & $3(2)$ \\
\hline $\mathrm{N}(1)$ & $25(2)$ & $35(2)$ & $30(2)$ & $3(2)$ & $9(2)$ & $5(2)$ \\
\hline $\mathrm{N}(2)$ & $22(2)$ & $27(2)$ & $24(2)$ & $2(2)$ & $5(2)$ & $0(2)$ \\
\hline $\mathrm{N}(3)$ & $23(2)$ & $25(2)$ & $27(2)$ & $6(2)$ & $8(2)$ & $3(2)$ \\
\hline $\mathrm{C}(1)$ & $32(3)$ & $57(3)$ & $41(3)$ & $7(3)$ & $11(3)$ & $6(3)$ \\
\hline$C(2)$ & $27(3)$ & $76(4)$ & $49(4)$ & $11(3)$ & $17(3)$ & $10(3)$ \\
\hline$C(3)$ & $43(4)$ & $71(4)$ & $59(4)$ & $21(3)$ & $25(3)$ & $-2(3)$ \\
\hline$C(4)$ & $30(3)$ & $54(3)$ & $50(4)$ & $14(3)$ & $14(3)$ & $1(3)$ \\
\hline$C(5)$ & $30(3)$ & $35(3)$ & $26(3)$ & $-1(2)$ & $8(2)$ & $-1(2)$ \\
\hline$C(6)$ & $19(3)$ & $30(2)$ & $21(2)$ & $2(2)$ & $5(2)$ & $2(2)$ \\
\hline$C(7)$ & $23(3)$ & $25(2)$ & $25(3)$ & $-2(2)$ & $4(2)$ & $5(2)$ \\
\hline$C(8)$ & $28(3)$ & $24(2)$ & $24(3)$ & $-4(2)$ & $3(2)$ & $2(2)$ \\
\hline $\mathrm{C}(11)$ & $33(3)$ & $30(3)$ & $53(4)$ & $10(3)$ & $10(3)$ & $-2(2)$ \\
\hline$C(12)$ & $34(3)$ & $29(2)$ & $42(3)$ & $-6(2)$ & $7(3)$ & $2(2)$ \\
\hline$C(13)$ & $22(3)$ & $25(2)$ & $29(3)$ & $9(2)$ & $5(2)$ & $4(2)$ \\
\hline$C(14)$ & $31(3)$ & $32(3)$ & $37(3)$ & $4(2)$ & $13(2)$ & $4(2)$ \\
\hline$C(17)$ & $40(3)$ & $30(3)$ & $43(3)$ & $7(2)$ & $18(3)$ & $8(2)$ \\
\hline$C(18)$ & $39(3)$ & $29(2)$ & $28(3)$ & $-2(2)$ & $6(2)$ & $0(2)$ \\
\hline$C(19)$ & $25(3)$ & $28(2)$ & $23(3)$ & $6(2)$ & $7(2)$ & $6(2)$ \\
\hline
\end{tabular}




\begin{tabular}{llllccc}
$\mathrm{C}(20)$ & $21(3)$ & $31(3)$ & $39(3)$ & $1(2)$ & $9(2)$ & $3(2)$ \\
$\mathrm{C}(21)$ & $39(3)$ & $35(3)$ & $51(3)$ & $-7(3)$ & $10(3)$ & $-4(2)$ \\
$\mathrm{C}(23)$ & $25(3)$ & $33(3)$ & $54(4)$ & $1(3)$ & $7(3)$ & $6(2)$ \\
\hline
\end{tabular}


Table S5. Hydrogen coordinates $\left(\times 10^{4}\right)$ and isotropic displacement parameters $\left(\AA^{2} \times 10^{3}\right)$ for complex 3.

\begin{tabular}{lrrrr}
\hline & $\mathrm{x}$ & $\mathrm{y}$ & $\mathrm{z}$ & $\mathrm{U}(\mathrm{eq})$ \\
\hline $\mathrm{H}(9 \mathrm{~A})$ & 2282 & -581 & 10670 & 66 \\
$\mathrm{H}(9 \mathrm{~B})$ & 2887 & -1005 & 10645 & 66 \\
$\mathrm{H}(9 \mathrm{C})$ & 2277 & -1308 & 9931 & 66 \\
$\mathrm{H}(10 \mathrm{~A})$ & 3731 & 3817 & 7559 & 120 \\
$\mathrm{H}(10 \mathrm{~B})$ & 4393 & 3581 & 8140 & 120 \\
$\mathrm{H}(10 \mathrm{C})$ & 3931 & 3860 & 8651 & 120 \\
$\mathrm{H}(15 \mathrm{~A})$ & 3966 & -235 & 8010 & 82 \\
$\mathrm{H}(15 \mathrm{~B})$ & 3686 & 54 & 6966 & 82 \\
$\mathrm{H}(15 \mathrm{C})$ & 3267 & -217 & 7553 & 82 \\
$\mathrm{H}(16 \mathrm{~A})$ & 4660 & -2630 & 11074 & 83 \\
$\mathrm{H}(16 \mathrm{~B})$ & 4911 & -2460 & 10238 & 83 \\
$\mathrm{H}(16 \mathrm{C})$ & 4260 & -2834 & 10051 & 83 \\
$\mathrm{H}(1)$ & 481 & 1910 & 8280 & 52 \\
$\mathrm{H}(2)$ & -83 & 956 & 8832 & 59 \\
$\mathrm{H}(3)$ & 345 & -336 & 9533 & 67 \\
$\mathrm{H}(4)$ & 1337 & -628 & 9686 & 54 \\
$\mathrm{H}(12)$ & 3301 & 2574 & 9032 & 44 \\
$\mathrm{H}(18)$ & 3454 & -1629 & 9540 & 39 \\
$\mathrm{H}(21 \mathrm{~A})$ & 4476 & 1307 & 10325 & 64 \\
$\mathrm{H}(21 B)$ & 4477 & 1105 & 11345 & 64 \\
$\mathrm{H}(21 \mathrm{C})$ & 3873 & 1327 & 10570 & 64 \\
\hline
\end{tabular}

